MODELING, IDENTIFICATION AND CONTROL, 1990, VOL. 11, NO. 2, 123-125

doi:10.4173/mic.1990.25

\title{
A note on a necessary condition for optimality
}

\section{DAVID DI RUSCIO†}

Keywords: Linear optimal control, multivariable systems, pole placements, eigenvalues, control theory.

A necessary condition is derived for the optimality of a linear multivariable feedback control system with respect to a quadratic performance index of infinite settling time.

\section{Introduction}

In linear quadratic system design the relation between the quadratic weights and the dynamic characteristics of the closed-loop system is of interest. This problem has been solved for a second order system (Di Ruscio and Balchen, 1990). In the general case, very little is known about these relations.

Let $\lambda_{i}, i=1, \ldots, n$ be the open-loop poles and $s_{i}, i=1, \ldots, n$ be the closed-loop poles. Mac Farlane (1970) presented an inequality between the product of the open-loop poles and the product of the closed-loop poles,

$$
\prod_{i=1}^{n}\left|s_{i}\right| \geqslant \prod_{i=1}^{n}\left|\lambda_{i}\right|
$$

Koussiouris (1982) (later corrected by Amin, 1984) presented an inequality between the sum of squares of the open-loop poles and closed-loop system poles,

$$
\sum_{i=1}^{n} s_{i}^{2} \geqslant \sum_{i=1}^{n} \lambda_{i}^{2}
$$

These relations are, as we can see, conservative because the quadratic weights do not appear in the relations. In this paper an exact relation between the open-loop and closed-loop system poles is presented. We will show that the relation by Amin and Koussiouris is a special case of our relation.

\section{Theory}

Let $\mathbf{A}$ be the $n \times n$ real state system matrix and $\mathrm{B}$ the $n \times r$ real control input matrix. Let $\mathbf{Q}$ be the $n \times n$ real symmetric state weight matrix and $\mathbf{P}$ the $r \times r$ real symmetric positive definite control input weight matrix. Then we have the following theorem.

\section{Theorem}

If $s_{i}, i=1, \ldots, n$ are the closed-loop system poles and $\lambda_{i}, i=1, \ldots, n$ are the open-loop system poles, then $s_{i}, i=1, \ldots, n$ are related to $A$ or $\lambda_{i}, Q$ and the positive semidefinite matrix $H=B P^{-1} B^{\mathrm{T}}$ by the following equality,

$$
\sum_{i=1}^{n} s_{i}^{2}=\operatorname{tr}\left(A^{2}\right)+\operatorname{tr}(H Q)
$$

Received 1 August, 1990.

† Division of Engineering Cybernetics, Norwegian Institute of Technology, N-7034 Trondheim, Norway. 
or equivalently

$$
\sum_{i=1}^{n}\left\{\left(\operatorname{Re} s_{i}\right)^{2}-\left(\operatorname{Im} s_{i}\right)^{2}\right\}=\sum_{i=1}^{n}\left\{\left(\operatorname{Re} \lambda_{i}\right)^{2}-\left(\operatorname{Im} \lambda_{i}\right)^{2}\right\}+\operatorname{tr}(H Q)
$$

Proof

The Hamiltonian matrix is derived from optimal control theory by augmenting the co-states to the state space model. The Hamiltonian is

$$
F=\left[\begin{array}{rl}
A & -H \\
-Q & -A^{\mathrm{T}}
\end{array}\right] \in \mathbf{R}^{2 n \times 2 n}
$$

$F$ has $2 n$ eigenvalues ( $n$ stable and $n$ unstable) located symmetrically about the imaginary axis. The stable eigenvalues are identical to the eigenvalues of the closedloop system matrix $(A+B G)=(A-H R)$, where $G$ is the feedback matrix and $R$ the symmetric solution of the algebraic Riccati equation. This can be seen from the following similarity transformation

$$
\left[\begin{array}{ll}
I & 0 \\
R & I
\end{array}\right]^{-1}\left[\begin{array}{rl}
A & -H \\
-Q & -A^{\mathrm{T}}
\end{array}\right]\left[\begin{array}{ll}
I & 0 \\
R & I
\end{array}\right]=\left[\begin{array}{cc}
A-H R & -H \\
0 & -(A-H R)^{\mathrm{T}}
\end{array}\right]
$$

This means that the closed-loop eigenvalue spectrum can be derived from $\boldsymbol{F}$ without solving the algebraic Riccati equation.

Let $\rho(F)$ denote the spectrum (set of $2 n$ eigenvalues) of $F$. Then we have that

$$
\begin{aligned}
\rho_{i}(F) & =s_{i}, i=1, \ldots, 2 n \\
s_{i} & =-s_{n+i}, i=1, \ldots, n \\
\operatorname{tr}(F) & =\sum_{i=1}^{2 n} s_{i}=0
\end{aligned}
$$

We also have this

$$
\begin{aligned}
\rho_{i}\left(F^{2}\right) & =s_{i}^{2}, i=1, \ldots, 2 n \\
s_{i}^{2} & =s_{n+i}^{2}, i=1, \ldots, n \\
\operatorname{tr}\left(F^{2}\right) & =2 \sum_{i=1}^{n} s_{i}^{2}
\end{aligned}
$$

We now need an expression for $\operatorname{tr}\left(F^{2}\right)$. We have

$$
F^{2}=\left[\begin{array}{cc}
A^{2}+H Q & H A^{\mathrm{T}}-A H \\
A^{\mathrm{T}} Q-Q A & \left(A^{2}+H Q\right)^{\mathrm{T}}
\end{array}\right]
$$

From (11) we have

$$
\operatorname{tr}\left(F^{2}\right)=\operatorname{tr}\left(A^{2}+H Q\right)+\operatorname{tr}\left(\left(A^{2}+H Q\right)^{\mathrm{T}}\right)=2 \operatorname{tr}\left(A^{2}+H Q\right)
$$

From (10) and (12) we get

$$
\sum_{i=1}^{n} s_{i}^{2}=\operatorname{tr}\left(A^{2}\right)+\operatorname{tr}(H Q)
$$

and the theorem is proved. 
$Q$ can be written as $Q=S^{\mathrm{T}} S(Q \geqslant 0$ and $\operatorname{rank}(S)=\operatorname{rank}(Q))$. Then we have that

$$
\operatorname{tr}(H Q)=\operatorname{tr}\left(H S^{\mathrm{T}} S\right)=\operatorname{tr}\left(S H S^{\mathrm{T}}\right) \geqslant 0
$$

because the matrix $S H S^{\mathrm{T}}$ is symmetric and positive semidefinite. Combining (2) and (14) we deduce the inequality presented in Amin (1984) and partly in Koussiouris (1982), i.e.

$$
\sum_{i=1}^{n}\left\{\left(\operatorname{Re} s_{i}\right)^{2}-\left(\operatorname{Im~} s_{i}\right)^{2}\right\} \geqslant \sum_{i=1}^{n}\left\{\left(\operatorname{Re} \lambda_{i}\right)^{2}-\left(\operatorname{Im} \lambda_{i}\right)^{2}\right\}
$$

An alternative way to establish the theorem is as follows. We have

$$
(A-H R)^{2}=(A-H R)(A-H R)=A^{2}+H(-R A+R H R)-A H R
$$

Combining equation (16) and the algebraic Riccati equation gives

$$
(A-H R)^{2}=A^{2}+H Q+\left(H A^{\mathrm{T}}-A H\right) R
$$

Take the trace on both sides of equation (17), note that $\operatorname{tr}\left[\left(H A^{\mathrm{T}}-A H\right) R\right]=0$, and equation (1) is proved.

The same procedure as above can be used to determine expressions for

$$
\sum_{i=1}^{n} s_{i}^{2 k}, k=1,2,3, \ldots
$$

For $k=2$ we have

$$
\begin{aligned}
\sum_{i=1}^{n} s_{i}^{4} & =\sum_{i=1}^{n}\left\{\left(\left(\operatorname{Re} s_{i}\right)^{2}-\left(\operatorname{Im} s_{i}\right)^{2}\right)^{2}-4\left(\operatorname{Re} s_{i}\right)^{2}\left(\operatorname{Im} s_{i}\right)^{2}\right\} \\
& =\operatorname{tr}\left(A^{4}+4 Q A^{2} H-2 Q A H A^{\mathrm{T}}+(H Q)^{2}\right) \\
& =\operatorname{tr}\left[\left(A^{2}+H Q\right)\left(A^{2}+H Q\right)+\left(A^{\mathrm{T}} Q-Q A\right)\left(H A^{\mathrm{T}}-A H\right)\right]
\end{aligned}
$$

Finally, note that this procedure cannot be used to determine expressions for

$$
\sum_{i=1}^{n} s_{i}^{2 k-1}, k=1,2,3, \ldots
$$

because

$$
\operatorname{tr}\left(F^{2 k-1}\right)=0, k=1,2,3, \ldots
$$

\section{Conclusions}

A necessary condition is presented for the optimality of a linear multivariable feedback control system with respect to a quadratic performance index of infinite settling time.

\section{REFERENCES}

AmIN, M. A. (1984). Further comments on 'A necessary condition for optimization in the frequency domain' and on 'Optimization and pole placement for a single input controllable system'. International Journal of Control, 40, 863-865.

Di Ruscio, D., and Balchen, J. G. (1990). A Schur method for designing LQ-optimal systems with prescribed eigenvalues. Modeling, Identification and Control, 11, 55-72.

Koussiouris, T. G. (1982). A necessary condition for optimization in the frequency domain. International Journal of Control, 36, 213-215.

Mac Farlane, A. G. J. (1970). Two necessary conditions in the frequency domain for the optimality of a multiple-input linear control system. Proceedings IEEE, 117, 464- 466. 